\begin{tabular}{|c|l|}
\hline Title & Evaluation of floating impeller phenomena in a Gyro centrifugal pump \\
\hline Author(s) & $\begin{array}{l}\text { Nishimura, Ikuy a; Ichikawa, S.; Mikami, M.; I shitoya, H.; Motomura, T.; Kawamura, M.; Linneweber, J.; Glueck, J.; } \\
\text { Shinohara, T.; Nose, Y. }\end{array}$ \\
\hline Citation & $\begin{array}{l}\text { Bio-Medical Materials and Engineering, 23(1-2), 49-55 } \\
\text { https://doi.org/L0.3233/BME-120731 }\end{array}$ \\
\hline Issue Date & 2013 \\
\hline Doc URL & http://hdl.handle.net/2115/52652 \\
\hline Type & article (author version) \\
\hline File Information & Nishimura.pdf \\
\hline
\end{tabular}

Instructions for use 


\section{Evaluation of Floating Impeller Phenomena in a Gyro Centrifugal Pump}

I.Nishimura, S.Ichikawa, M.Mikami, H.Ishitoya, T.Motomura, M.Kawamura, J.Linneweber, J.Glueck, T.Shinohara, and Y.Nosé

Michael E. DeBakey Department of Surgery, Baylor College of Medicine, Houston, Texas, USA

Ikuya Nishimura, Ph.D.

Hokkaido University, Graduate School of Engineering, Division of Systems and Information Engineering, Department of Biophysical Engineering.

N13 W8, Kita-ku, Sapporo 060-8628, Hokkaido, Japan.

Tel/Fax: +81-11-706-6585 (direct) E-mail: mura@bme.eng.hokudai.ac.jp 
Abstract: The Gyro centrifugal Pump developed as a totally implantable artificial heart was designed with a free impeller, in which the rotational shaft (male bearing) of the impeller was completely separated from the female bearing. For this type of pump, it is very important to keep the proper magnet balance (impeller-magnet and actuator-magnet) in order to prevent thrombus formation and/or bearing wear. When the magnet balance is not proper, the impeller is jerked down into the bottom bearing. On the other hand, if magnet balance is proper, the impeller lifted off the bottom of the pump housing within a certain range of pumping conditions. In this study, this floating phenomenon was investigated in detail. The floating phenomenon was proved by observation of the impeller behavior using a transparent acrylic pump. The impeller floating phenomenon was mapped on a pump performance curve. The impeller floating phenomenon is affected by the magnet-magnet coupling distance and rotational speed of the impeller. In order to keep the proper magnet balance and to maintain the impeller floating phenomenon at the driving condition of right and left pump, the magnet-magnet coupling distance was altered by a spacer which was installed between the pump and actuator. It became clear that the same pump could handle different conditions (right and left ventricular assist), by just changing the thickness of the spacer. When magnet balance is proper, the floating impeller phenomenon occurs automatically in response to the impeller rev. It is called "the dynamic RPM suspension".

Key Word: Free Impeller Gyro Pump, Pivot bearing, Impeller floating phenomenon, Magnet coupling distance, the dynamic RPM suspension. 


\section{Introduction}

The magnet-magnet coupling system is a very important aspect of a centrifugal pump in regards to the thrombus formation or hemolysis, and bearing wear (1-2). A strong magnet force jerks the impeller down into the bottom bearing and results in thrombus formation or bearing wear. Conversely, a weak magnet force results in decoupling the combination of impeller and actuator, and decline of efficiency. Consequently, keeping the proper magnet balance is very important for a long-term clinically applied centrifugal pump.

The impeller floating phenomenon can be observed in a pivot bearing supported pump (3-4). It is considered that the condition in which the impeller floating phenomenon occurs is the proper magnet balance. This phenomenon is affected by the magnet (impeller)-magnet (actuator) coupling distance and rotational speed of the impeller. The aim of this study is to map the impeller floating phenomenon on a pump performance curve in order to maintain the proper magnet balance during pumping.

\section{Materials and Methods}

A completely seal-less Gyro centrifugal pump was used in this study. This pump, PI (permanently implantable) series, is being developed as a totally implantable artificial heart (5) (6). This pump is composed of the housing, impeller, and actuator. The pump has ultra-high molecular weight polyethylene female bearings and alumina ceramics male bearing. Six strong Neodymium-iron-boron composite magnets (diameter: 10mm) are installed inside the impeller and 
actuator. The direct current (DC) brushless motor (Softronics Co.) was used to drive the pump. The combination of impeller (magnetic flux: $3.4 \mathrm{KG}$, at the surface of the impeller) and actuator (magnetic flux: $2.36 \mathrm{KG}$, at the surface of the actuator), and the combination of impeller (magnetic flux: $3.3 \mathrm{KG}$, at the surface of the impeller) and actuator (magnetic flux: $2.12 \mathrm{KG}$, at the surface of the actuator) were used for this study. This study used an acrylic pump, which was identical to the PI pump, to observe the impeller floating phenomenon. The schematic figure of the experimental setup is shown in Fig. 1. These studies were performed with a glycerin/water $(37 \mathrm{wt} \%)$ solution. In order to observe the impeller floating phenomenon, the distance between the top and bottom female bearing was lengthened by $1 \mathrm{~mm}$. The minimal magnet coupling distance (MCD, see Fig.1) between the impeller magnet and actuator magnets was designed to be $9 \mathrm{~mm}$. The MCD was altered from $9 \mathrm{~mm}$ (standard condition) to $12.5 \mathrm{~mm}$ by an acrylic spacer which was installed between the pump and actuator. The floating phenomenon was observed in Fig.2. Also, Fig.2 (a) demonstrates that the impeller male bearing comes into contact with the bottom female bearing (bottom contact mode). Fig.2(b) demonstrates that the impeller male bearing is apart from the bottom female bearing and comes into contact with the top female bearing (top contact mode). These authors investigated the relationship between pump flow and pressure as a parameter of the impeller rotational speed (pump performance curve). The impeller floating phenomenon was mapped on the pump performance curve. 


\section{Results}

Fig.3 shows the results using a combination of impeller (magnetic flux: $3.4 \mathrm{KG}$ ) and actuator (magnetic flux: 2.36KG). As indicated in Fig.3, the results were plotted on a Flow-Pressure curve. Fig.3 illustrates top contact area, top contact point, hysteresis area, bottom contact point, and bottom contact area, respectively. The MCD was altered from $9 \mathrm{~mm}$ (standard condition) to $12.5 \mathrm{~mm}$ by spacer. At a low impeller rotational speed, the impeller position was in the bottom contact mode. When the rotational speed was increased, impeller floating phenomenon appeared at the top contact point. And at a high impeller rotational speed, the impeller position was in the top contact mode. When the rotational speed was decreased the impeller floating phenomenon disappeared at the bottom contact point. The area surrounded by the top contact point and bottom contact point was the hysteresis area. In this area, the magnetic forces that jerks the impeller down into the bottom contact mode and hydromechanical forces which makes the impeller float balance. As indicated in Fig.3 (a) (MCD=9mm) through Fig.3 (f) $(\mathrm{MCD}=12.5 \mathrm{~mm})$, as the $\mathrm{MCD}$ increased, impeller rotational speed in which the floating phenomenon appeared decreased. Consequently, the top contact area was extended and the bottom contact area was reduced. However, the size of the hysteresis area remained unchanged. In MCD $=9 \mathrm{~mm}$ (Fig.3 (a)), the ordinary left ventricular assist device (LVAD) driving condition of $5 \mathrm{~L} / \mathrm{min}$ against $100 \mathrm{~mm} \mathrm{Hg}$ belonged to the hysteresis area. When $\mathrm{MCD}=11 \mathrm{~mm}$ or over, the LVAD driving condition belonged to the top contact area. Therefore, the proper spacers can be selected for the left pump $(2.5 \mathrm{~mm}$ spacer $(\mathrm{MCD}=11.5 \mathrm{~mm}))$ and for the right pump $(3 \mathrm{~mm}$ spacer $(\mathrm{MCD}=12 \mathrm{~mm}))$. 


\section{Discussion}

During pumping, the working forces on the impeller are magnetic forces caused by the magnetic coupling, and the hydromechanical forces caused by differential pressure. The magnetic force which jerks the impeller down into the bottom bearing is calculated as:

$$
\mathbf{F}=\frac{m_{1} \cdot m_{2}}{4 \cdot \pi \cdot \mu_{0} \cdot r^{2}}
$$

where $\mathrm{m} 1, \mathrm{~m} 2=$ magnetic flux, $\mu 0=$ magnetic permeability, $\mathrm{r}=$ magnet coupling distance (MCD) .

The attractive forces combination of the impeller (magnetic flux: $3.4 \mathrm{KG}$ ) and actuator (magnetic flux: $2.36 \mathrm{KG}$ ), and the combination of impeller (magnetic flux: $3.3 \mathrm{KG}$ ) and actuator (magnetic flux: 2.12KG) were estimated in Fig.4, respectively. When MCD increased, the force decreased. The force of the combination of impeller (magnetic flux: 3.4KG) and actuator (magnetic flux: 2.36KG) at $\mathrm{MCD}=12 \mathrm{~mm}$ is equal to the force of combination of impeller (magnetic flux: $3.3 \mathrm{KG}$ ) and actuator (magnetic flux: $2.12 \mathrm{KG}$ ) at $\mathrm{MCD}=11 \mathrm{~mm}$. Fig. 5 shows the measurement results for the impeller floating phenomenon in each condition. The top contact area, the hysteresis area, and the bottom contact area are located in almost the same position as shown in Fig.5 (a) and (b). The impeller position, namely the impeller floating phenomenon can be estimated using the measured magnetic forces and $\mathrm{MCD}$, and this phenomenon might be able to be regulated by altering the MCD. As this pivot bearing supported pump, the impeller position is very important. Durability of the ultra-high molecular weight polyethylene female bearing (1) is affected by the wear caused by an imbalance of the working forces on the impeller. In order to reduce the wear, the working forces 
on the impeller should be balanced and the friction between the male and female pivot bearing will decrease. When the impeller is in the top contact position, the clearance between impeller bottom and bottom housing increases. Therefore, blood flow in this clearance will be promoted (7), stagnant area of the pump will disappear, and a desirable condition for antithrombogenecity will be improved. Accordingly, the impeller position has to be in the top contact mode. However, driving condition of left pump is different from that of right pump. Therefore, two kinds of pumps are generally necessary for total BiVAD artificial heart implantation. However, a same pump is useful in a variety of conditions (right and left ventricular assist), just changing the thickness of the spacer. When the pump is maintained the proper magnet balance condition, the floating impeller phenomenon occurs automatically in response to the impeller rev. It is called "the dynamic RPM suspension".

\section{Conclusion}

Keeping the proper MCD and maintain the impeller position as the top contact mode is very important for a long-term clinically applied centrifugal pump. The impeller position (top contact mode, or bottom contact mode) is very important for a pivot bearing supported centrifugal pump in regards to the thrombus formation or hemolysis, and bearing wear. In this study, the impeller floating phenomenon was investigated in detail, and we indicated that the impeller position was regulated by changing the MCD by spacer. It became clear that the impeller floating phenomenon was possible to be estimated and the same pump could cope with the different conditions (right 
and left ventricular assist), just by changing the thickness of the spacer.

\section{References}

1. T.Nakazawa, K.Makinouchi, Y.Takami, R.Benkowski, J.Glueck, S.Takatani, and Y.Nosé. The effect of impeller-driver magnet coupling distance on hemolysis in a compact centrifugal pump. Artif Organs 1996;20:252-257

2. K.Makinouchi, T.Nakazawa, Y.Takami, S.Takatani, and Y.Nosé. Evaluation of the wear of the pivot bearing in the Gyro C1E3 pump. Artif Organs 1996;20:523-528

3. T.Nakazawa, Y.Takami, K.Makinouchi, R.Benkowski, J.Glueck, G.Damm, and Y.Nosé. Hydraulic assessment of the floating impeller phenomena in a centrifugal pump. Artif Organs $1997 ; 21: 78-82$

4. Y.Ohara, S.Takatani and Y.Nosé. In vitro analysis of the floating mode phenomenon in the free impeller Gyro pump. Artif Organs 1995;19:635-638

5. Y.Yoshikawa, K.Nonaka, J.Linneweber, S.Kawahito, G.Ohtsuka, K.Nakata, T.Takano, S.Schulte-Eistrup, J.Glueck, H.Schima, E.Wolner, and Y.Nosé. Development of the NEDO implantable ventricular assist device with Gyro centrifugal pump. Artif Organs 2000;24:459-467

6. Y.Takami, A.Andrade, T.Nakazawa, K.Makinouchi, J.Glueck, R.Benkowski, Y.Nosé. Eccentric inlet port of the pivot bearing supported Gyro centrifugal pump. Artif Organs 1997;21:312-317

7. Y.Ohara, M.Murase, Y.Nosé. Hemolytic effect of the secondary vane incorporated into the back side of the impeller. Artif Organs 1997;21:694-699 
Ikuya Nishimura

Figure 1

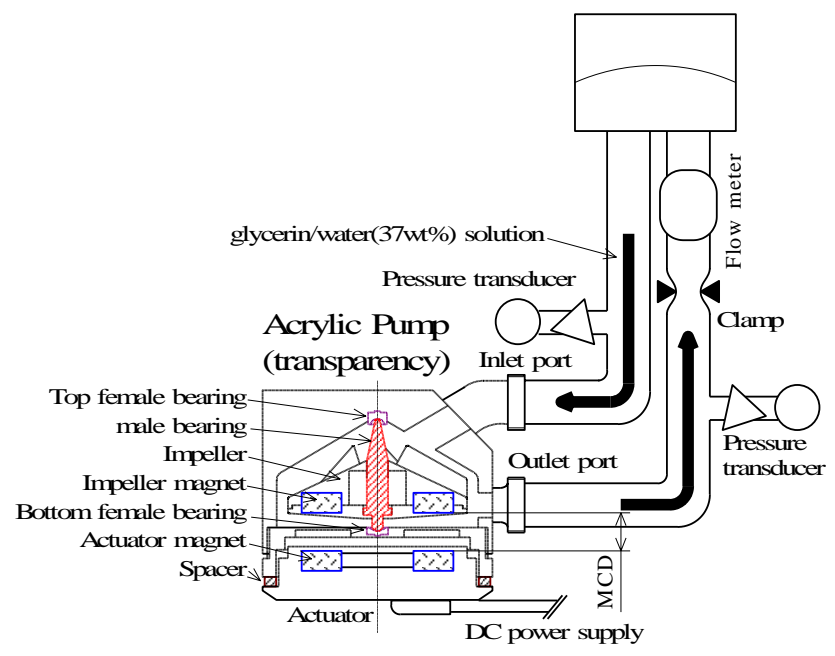


Ikuya Nishimura

Figure 2

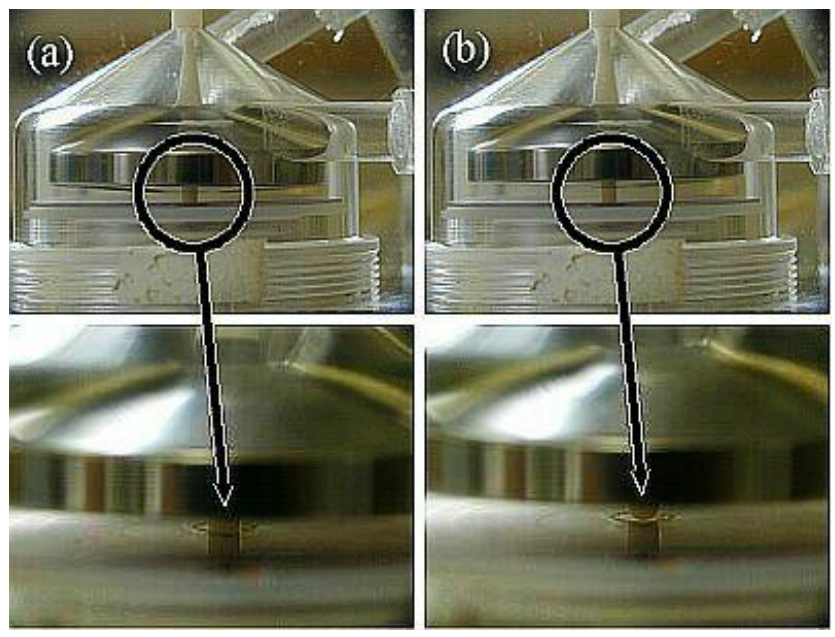


Ikuya Nishimura

Figure 3

(a) $\mathrm{MCD}=9 \mathrm{~mm}$
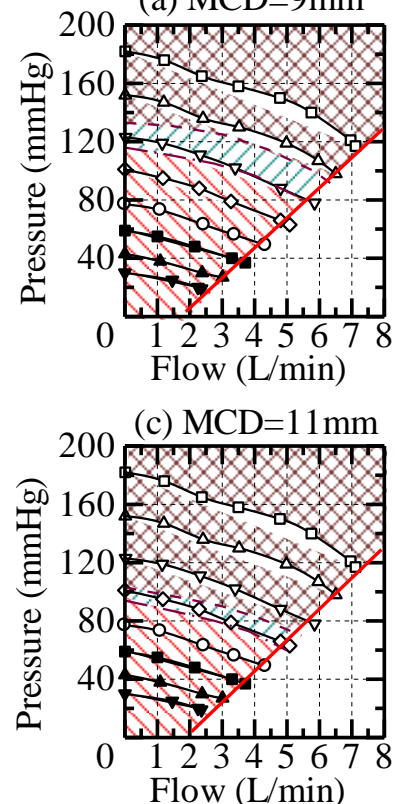

(e) $\mathrm{MCD}=12 \mathrm{~mm}$

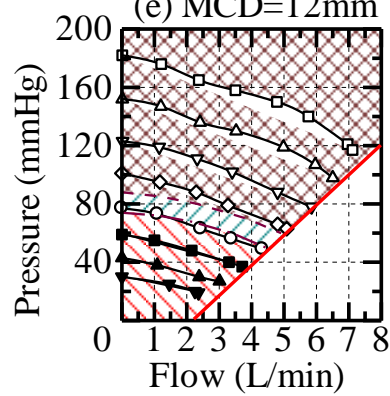

$\longrightarrow$ 2400RPM

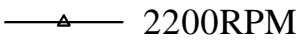

$\longrightarrow \quad$ 2000RPM

$\rightarrow \quad 1800 \mathrm{RPM}$

—_ 1600RPM

$\longrightarrow$ 1400RPM

$\longrightarrow$ 1200RPM

$\longrightarrow$ 1000RPM (b) $\mathrm{MCD}=10 \mathrm{~mm}$
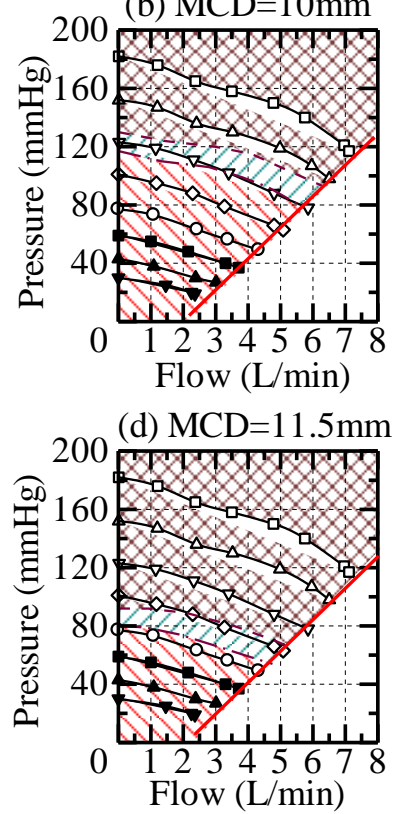

(f) $\mathrm{MCD}=12.5 \mathrm{~mm}$

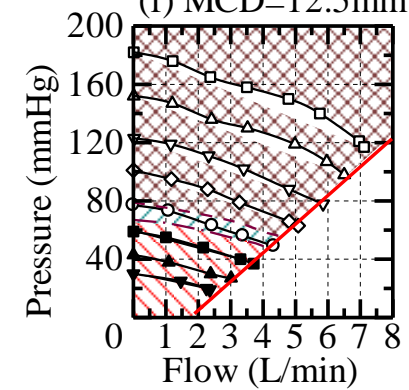

Top contact area

Top contact point

Hysteresis area

-.-... Bottom contact point Bottom contact area 
Ikuya Nishimura

Figure 4

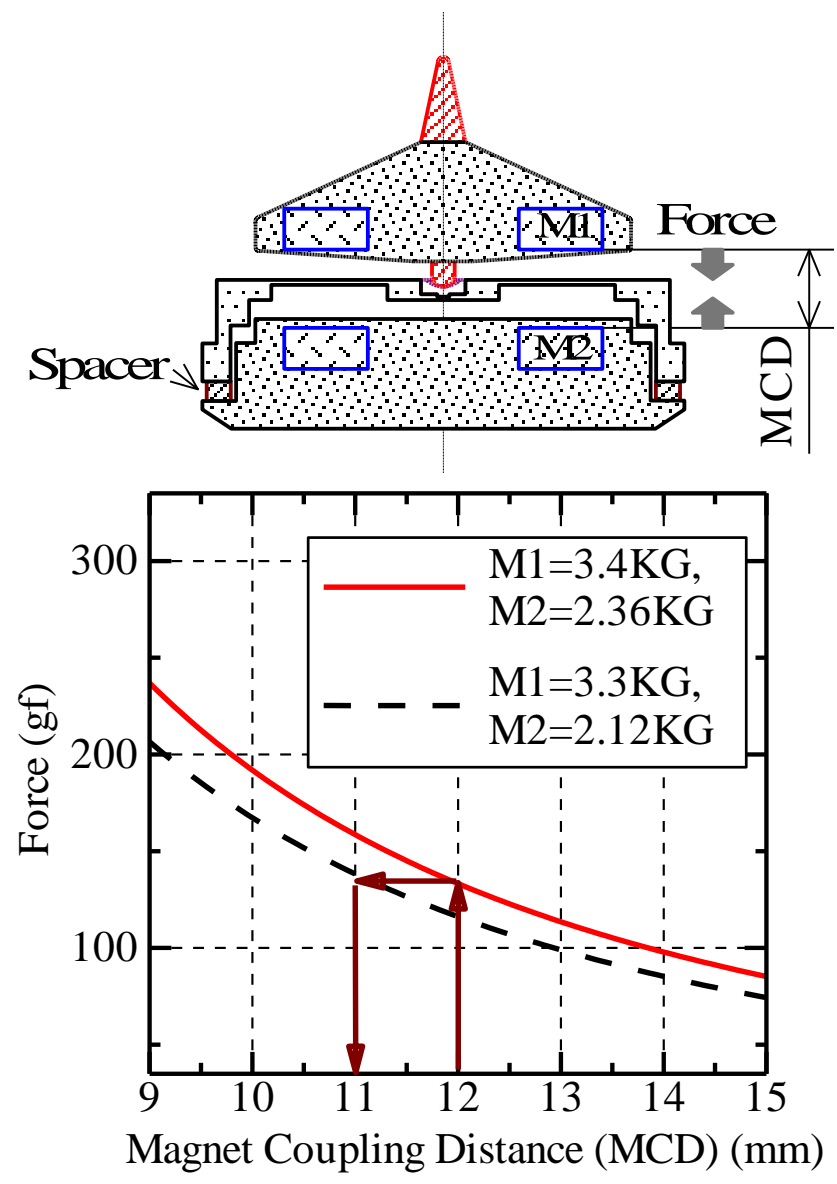


Ikuya Nishimura

Figure 5
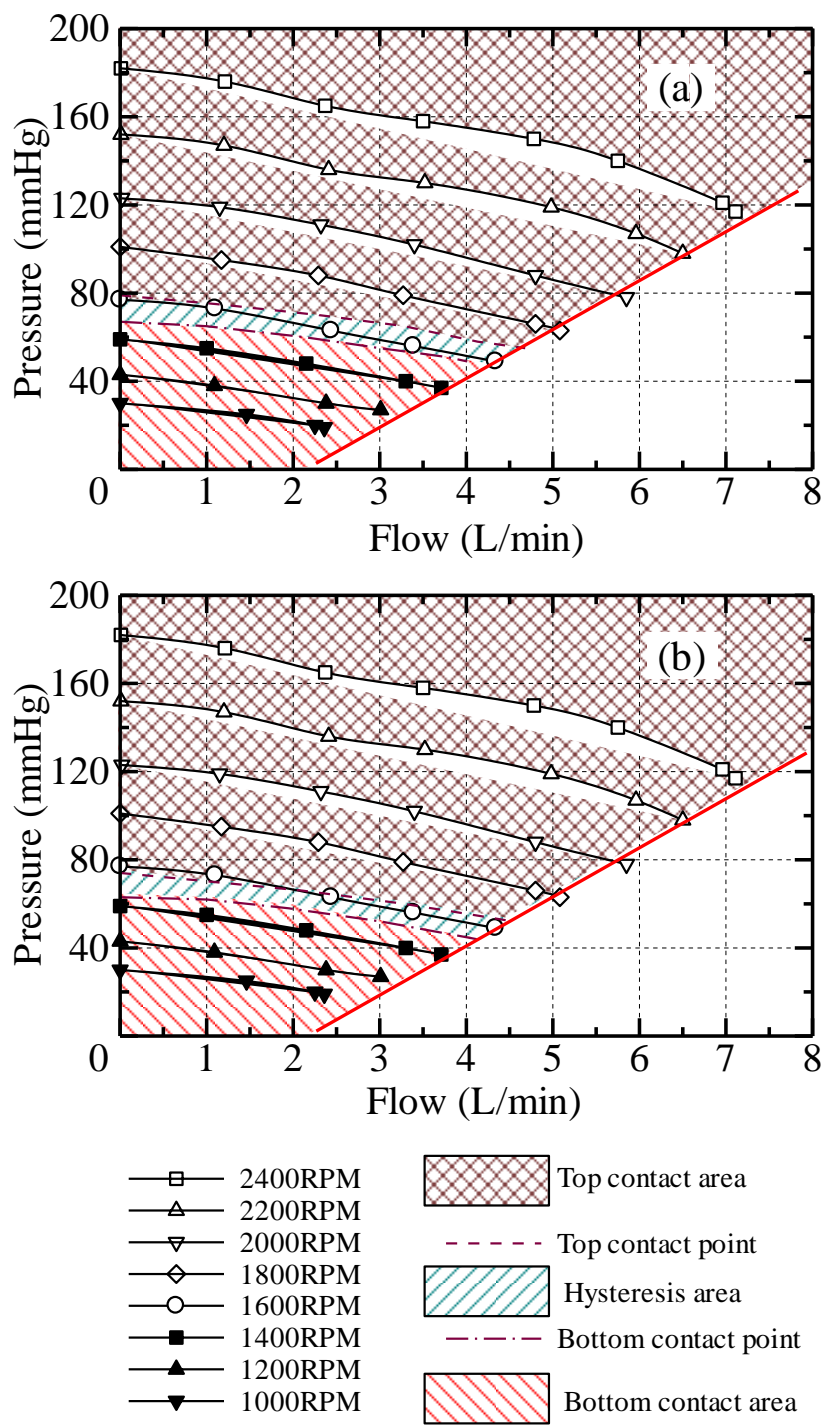
Ikuya Nishimura

Fig. 1. This schematic shows the experimental setup to observe the impeller position.

Fig. 2. Impeller position. (a)Bottom contact (b)Top contact

Fig. 3. Mapping of the impeller floating phenomenon on a pump performance curve. The combination of impeller (magnetic flux: $3.4 \mathrm{KG}$ ) and actuator (magnetic flux: $2.36 \mathrm{KG}$ )

Fig. 4. Estimation of the attractive forces with combination of impeller and actuator.

Fig. 5. Comparison of the impeller floating phenomenon.

(a) Combination of impeller (magnetic flux : $3.4 \mathrm{KG}$ ) and actuator (magnetic flux : $2.36 \mathrm{KG}$ ) at $\mathrm{MCD}=12 \mathrm{~mm}$

(b) Combination of impeller (magnetic flux : $3.3 \mathrm{KG}$ ) and actuator (magnetic flux : $2.12 \mathrm{KG}$ ) at $\mathrm{MCD}=11 \mathrm{~mm}$ 